Musées, Patrimoine et Culture scientifiques et techniques

$181 \mid 2019$

janvier-février 2019

\title{
La documentation dans l'organisation muséale. Approches épistémologiques
}

\section{Gérard Régimbeau}

\section{OpenEdition}

\section{Journals}

Édition électronique

URL : http://journals.openedition.org/ocim/2247

DOI : $10.4000 /$ ocim. 2247

ISSN : 2108-646X

Éditeur

OCIM

Édition imprimée

Date de publication : 1 janvier 2019

Pagination : 38-45

ISSN : 0994-1908

Référence électronique

Gérard Régimbeau, «La documentation dans l'organisation muséale. Approches épistémologiques », La Lettre de I'OCIM [En ligne], 181 | 2019, mis en ligne le 01 janvier 2020, consulté le 16 mars 2020 URL : http://journals.openedition.org/ocim/2247 ; DOI : https://doi.org/10.4000/ocim.2247

Ce document a été généré automatiquement le 16 mars 2020.

Tous droits réservés 


\section{La documentation dans}

l'organisation muséale. Approches épistémologiques

Gérard Régimbeau

La Bibliothèque Cartailhac du muséum d'Histoire naturelle de Toulouse

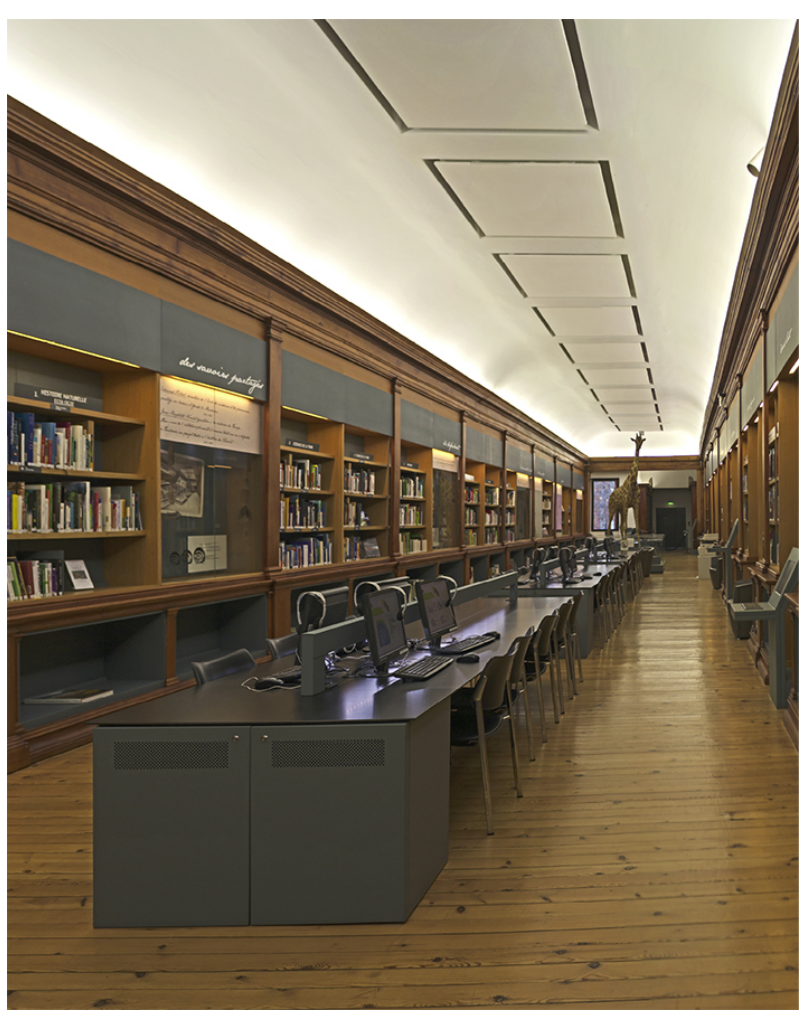

(c) Didier Descouens/Wikimédia Commons 
1 Trois points particuliers sont retenus sur le thème de la capitalisation des ressources documentaires selon les logiques internes aux structures ou les nouvelles logiques induites par les données massives et l'ouverture des accès : - le caractère organique de la documentation en contexte muséal ;

2 - la tendance croissante au partage des documents culturels et scientifiques ;

3 - la pérennité du traitement et de la diffusion documentaires dans les politiques muséales.

4 L'approche épistémologique de ces trois notions : organicité, partage et pérennité de l'information amènent à des réflexions situant leur intérêt conjoncturel à un stade donné de la muséologie.

5 Une question épistémologique ne passe pas par une mise à l'écart de la pratique, mais au contraire invite celle-ci à faire reposer l'analyse sur plusieurs pieds. La documentation muséale s'est bâtie en permanence dans la praxis et l'empirie autant que par des préceptes et des concepts. Ce qui signifie la compréhension des liens avec la structure ou l'organisme qui l'accueille et l'utilise, de son rôle d'outil, du modèle théorique qui en résulte et de sa fonction structurelle dans une approche globale. Si l'on se réfère à certains indicateurs de la littérature muséologique de ces vingt dernières années, la documentation y prend une place grandissante mais pas encore totalement reconnue dans la diversité et l'ampleur de ses objets.

\section{Organicité des ressources documentaires}

6 Si l'on en croit Geoffrey Lewis dans Comment gérer un musée pour la partie « Histoire de l'activité de collecte » (Lewis, 2006,) : "Le concept de musée apparaît [...] au début du II millénaire av. J.C. à Larsa, en Mésopotamie, où les écoles reproduisent les copies d'anciennes inscriptions à des fins pédagogiques. Les témoignages archéologiques du VIe siècle av. J.-C. conservés à Ur, suggèrent [...] une collection d'antiquités dans une salle près de l'école du temple, avec une plaque décrivant des inscriptions de briques trouvées sur place, probablement emblématique d'une "marque de musée" ". Autre indicateur attesté par Alain Schnapp à propos de l'Égypte antique : «Les scribes ont été les premiers à découvrir les rapports entre monument et texte, entre données matérielles et données textuelles. Du monde grécoromain au Moyen Âge, de la Renaissance à l'âge de Raison, les hommes du texte et les hommes del'objet [sic !], philologues et antiquaires ont entretenu uncontinuel dialogue, sur les méthodes, les moyens et les fins de la collection » (Schnapp, 2003). Ainsi, dès les premiers témoins de collections dans l'Antiquité, on rencontre des traces documentaires associées aux objets témoignant d'une activité de collecte ou d'inventaire. Partie constitutive et constituante de leurs missions, on ne peut donc éviter cette part de la ressource documentaire, incessible et évolutive, dans le système global des lieux et institutions voués aux collections.

7 Avec le dossier Culture \& Musées $\left(n^{\circ} 22,2013\right)$ intitulé «Documenter les collections, cataloguer l'exposition ", qui avait pour objectif de rassembler des enquêtes et des réflexions actuelles sur la documentation en tant qu'objet d'étude non seulement sous ses aspects techniques mais aussi communicationnels en soulignant son apport à la médiation des savoirs, les contributions ont montré :

8 - l'impact du numérique sur l'élaboration des dossiers d'œuvres marquant une transition dans l'histoire des supports ; 
9 - les choix et l'organisation d'items ou d'objets dans la gestion pratique et symbolique du patrimoine mondial éclairant les moyens de légitimation des biens ;

10 - les effets sémiotiques de la réalisation d'un portail des témoins patrimoniaux d'une " bibliothèque numérique » engageant au décryptage d'un impensé de la compilation se substituant à la pensée de la collection ;

11 - la liaison de systèmes d'information par l'organisation ontologique des données grâce à l'application d'un Modèle de référence conceptuel (Conceptual Reference Model) permettant de restituer du sens à la formalisation des données dans l'organisation des savoirs ;

12 - la collecte de traces signifiantes ajoutant à la théorisation des implications mémorielles du document pour la compréhension et la transmission de la performance dans le cadre de sa muséalisation ou dans le cadre d'une constitution de ressources pour une histoire des expositions ${ }^{1}$.

La Bibliothèque du Rijksmuseum à Amsterdam

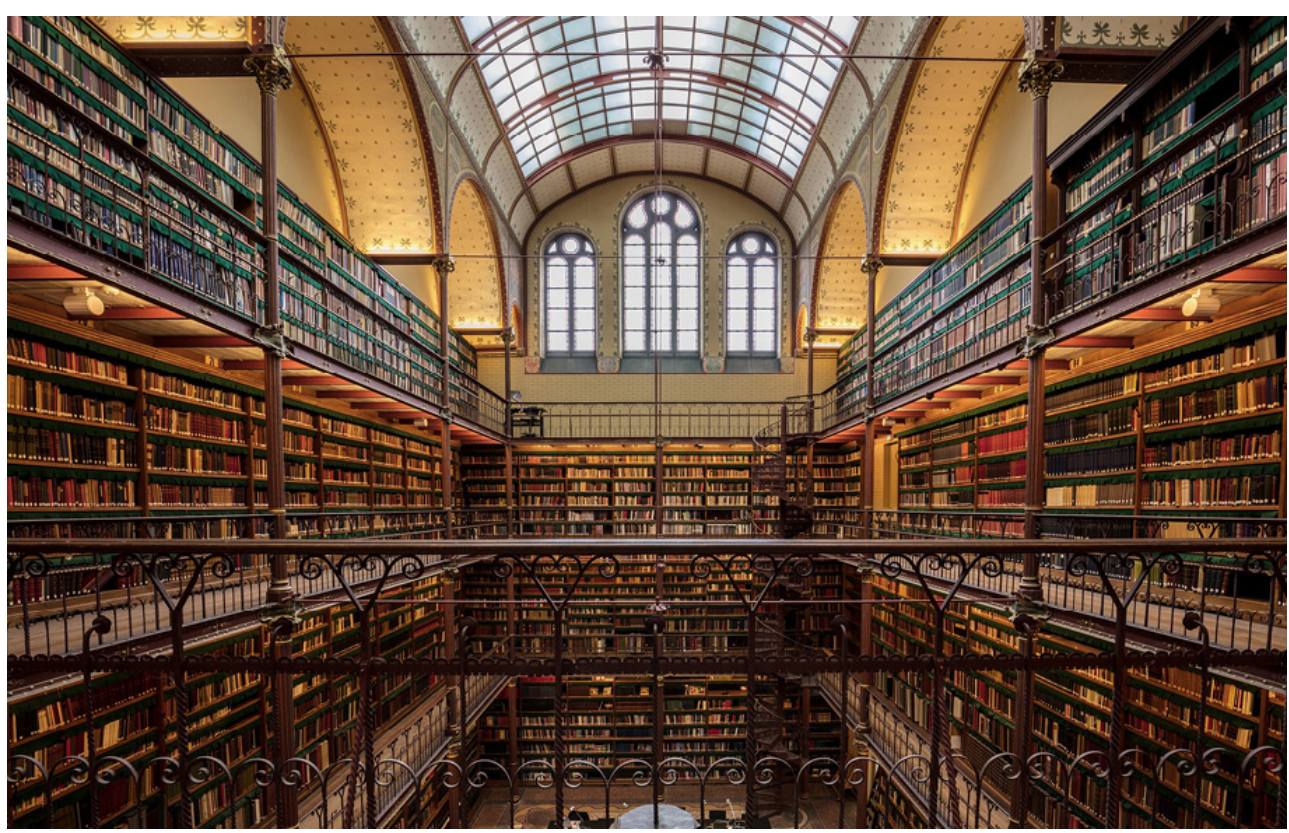

(C) Michael Beckwith

13 Des processus structurant la production documentaire en ressortent : notamment la patrimonialisation, la muséalisation, la normalisation et l'historicisation, qu'il serait possible de projeter sur d'autres configurations socio-historiques. Ces processus interviennent dans la construction mémorielle en impliquant chaque fois la documentation soit dans les données et métadonnées directes, soit dans la restitution éditoriale ou éditorialisée. Les études ont apporté sur ces différents plans des questionnements sur les changements de notre rapport au document, à ses contenus et à sa matérialité ; sur les fonctions légitimantes de l'inscription et de la trace ; sur l'harmonisation des principes d'une conception et d'une formalisation généalogique des métadonnées ; sur la réflexivité nécessaire au travail de mise en ligne des bibliothèques numériques; et sur le choix des témoins à conserver pour témoigner des activités de diffusion et d'exposition des objets et sujets muséaux. 
14 Pour ajouter à cette approche du champ théorique et pratique de la documentation muséale, il faut soulever une autre question qui concerne le statut même du document, car malgré son acception instable, celui-ci demeure un point essentiel où convergent muséologie et enjeux informationnels dans un contexte patrimonial ou de médiation des sciences. Il s'avère, d'une part, qu'un parti pris philosophique esthétique peut amener à considérer une pièce muséale comme une œuvre irréductible à toute désignation documentaire. Cette position peut, à son tour, entraîner des discussions cruciales sur le sens (historique, social, culturel) à donner à une collection et, de ce point de vue, les échanges qui eurent lieu au moment de la sélection des pièces du musée du Quai Branly à Paris (Sergent, 2007 ; Grognet, 2007) demeurent une matière à réflexion toujours active pour saisir les contradictions et les déplacements de question qui traversent le champ anthropologique. Il existe, d'autre part, selon le niveau de généralité ou de spécificité impliqué, une différence dans les critères qualifiant le document sous ses catégories primaire ou secondaire. En effet, il y a, d'une part, des pièces muséales considérées comme des documents primaires et les documents issus du traitement documentaire (cartel, fiche, notice, dossier...) de ces pièces qui sont dits " documents secondaires " en muséographie. Mais on sait, par ailleurs, que la terminologie documentaire ou bibliothéconomique distingue le document primaire ou document source (article, texte ou autre support d'un contenu premier) et le document secondaire comme une bibliographie à propos de ce document primaire. On perçoit donc que dans la documentation muséale, les termes recouvrent deux réalités différentes. Ces remarques ne s'imposaient pas quand la documentation restait en sousmain du traitement muséal mais, à l'heure actuelle, les principes taxinomiques doivent retrouver une cohérence dans une activité documentaire explicitée.

\section{Partage de données}

15 Aborder la question de la documentation muséale suppose aujourd'hui de la définir dans ses dimensions non seulement organiques mais aussi dans ses fonctions communicationnelles. La prise en charge de la production de données par des acteurs multiples et leur mise à disposition pourrait se rassembler sous la notion de " documents partagés ». Concernant la création et l'usage des documents, elle permet de mettre l'accent sur cette construction commune d'une information. La notion vaut pour de nombreuses fonctions et activités muséales : pour la gestion, la médiation, l'exposition ; dans les secteurs de l'étude, de la visite, de l'accueil. L'information déclinée en supports multiples intéresse maintenant, au-delà d'un objectif interne, la demande par le public d'une connaissance contextualisée des pièces ou des documents muséaux. On rejoint ici la question de la méta-documentation (Rizza, 2014).

De plus, il est difficile maintenant de n'envisager qu'une documentation des musées, car cette expression pourrait induire à restreindre le champ concerné. Il faut aussi faire mention d'une documentation au musée ou dans les musées pour mieux indiquer que son origine n'est pas seulement interne mais qu'elle y entre aussi, par acquisition, onéreuse ou gratuite, veille ou transfert, pour les besoins de différentes activités. Mobilisant les professionnels dans le cadre des travaux de renseignement des collections ou bien dans l'ouverture au public de bibliothèques, médiathèques et services dépendants, les fonds et collections documentaires nécessitent une gestion complémentaire et croisée à celle des données et métadonnées strictement muséales. 
Divers cartels issus du dossier d'œuvre du modèle La Muiron conservé au musée national de la Marine à Paris.

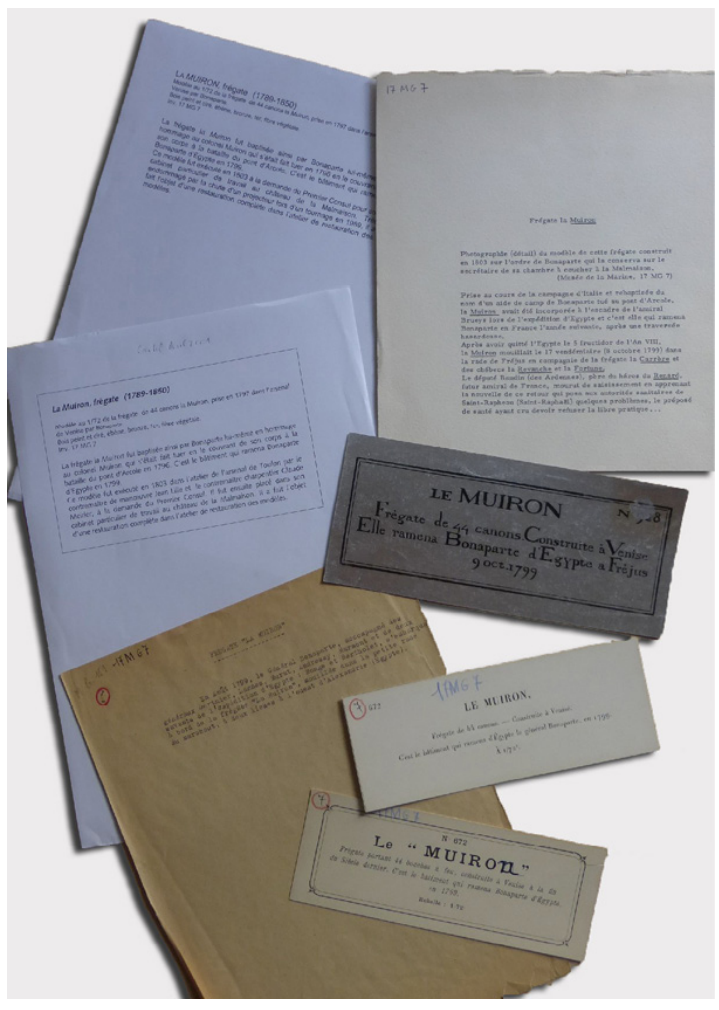

(c) Angelina Meslem

Le musée partage donc plusieurs fois les documents : en qualité d'auteur et en tant qu'éditeur ou diffuseur en les mettant à la disposition des citoyens, érudits ou néophytes. Sur ce point, il rencontre à nouveau la question de la vulgarisation, passant du document « intra-muséal » au document " extra » ou " épi-muséal » (adjectif construit sur « épitexte »), et ce, de manière gratuite ou payante vers des internautes et autres usagers. Enfin, il partage ceux qui proviennent de l'extérieur, en tant que lieu de savoir alimenté par d'autres ressources. Mais à cela, il faut ajouter une autre acception de « document partagé » en référence au document « mis en commun » des pratiques numériques collaboratives pour l'écriture ou la diffusion, et en référence à un document mis en ligne comme un post ou une vidéo déposée sur une plateforme.

Ouvert sur plusieurs modes de médiation, le musée a pour spécificité de répondre à des degrés de diffusion, de vulgarisation et de promotion pour lesquels il doit sans cesse réinvestir des matériaux intellectuels et sensibles sur les plans de la recherche et de l'administration, sur les plans de l'accueil, notamment de la visite, et de l'édition, sur les plans de la "médiation culturelle " et de l'événementiel. Comme il est dit de l'éducation ou d'une responsabilité sociale, que nous avons dans leur exercice des droits et des devoirs, l'idée de document partagé a ceci d'intéressant qu'elle met en relation les missions d'une institution avec des destinataires ou des usagers en intégrant aussi une forme de réciprocité. Les innovations dans les techniques d'enregistrement et de conservation que furent la photographie, le microfilmage, les supports magnétiques et la numérisation ont entraîné des changements dans la conception et la mise en forme des métadonnées. On en vient à cette implication des 
usagers dans une forme d'interactivité avec les producteurs de données et les documentalistes qui peut aider à comprendre ce que représente un travail scientifique muséal et à le soutenir (Régimbeau, 2014).

Les réserves du musée des Arts et Métiers à Paris

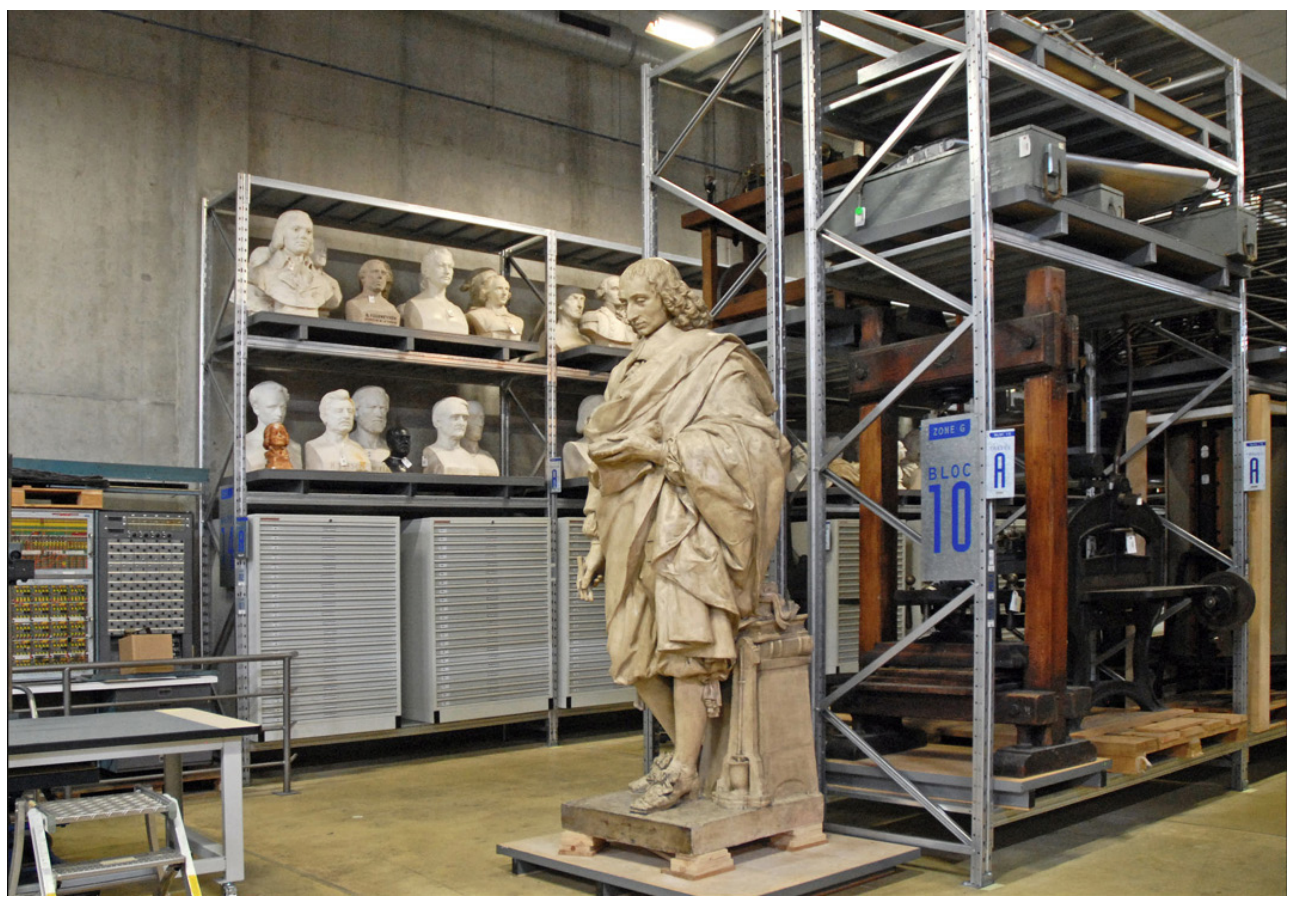

(c) Jean-Pierre Dalbéra/Wikimédia Commons

\section{Pérennité et médiation}

Si pour Jacques Theys, "l'ambiguité, on le sait, a depuisl'origine, été constitutive de l'émergence du concept de développement durable " et que ce dernier a tendance à être dépassé par des notions "comme "l'économie verte", "la transition écologique" (ou énergétique), "la résilience" ... ou "la décroissance" » (Theys, 2012), on reprendra cependant cette formule d'un point de vue critique en précisant qu'elle s'inscrit dans un cadre de projets de recherches et d'applications qui tente d'anticiper les enjeux, voire les formes d'un développement au regard de la pensée de la complexité (Morin, 1991).

La perspective documentaire ne peut ignorer que la notion de médiation prend place dans un ensemble, le musée, où le débat existe sur la notion même (Chaumier, 2008). On mentionnera seulement que l'étendue de son utilisation dans les sciences représente l'importance d'une mise au point sur les phénomènes de processus (chaînes, phases, ruptures, lieux, durées...) faisant intervenir divers acteurs et facteurs, humains et nonhumains, amenant à discerner des émergences plus que des causalités. Un des processus actuellement interrogé avec ou dans la médiation est donc celui de la durabilité, avec l'ensemble de la politique muséale. À l'instar de ce qui est mis en place dans les bibliothèques avec les critères qui définissent une politique documentaire (voir les travaux du Groupe PolDoc et notamment ceux de Jérôme Pouchol qui a dirigé en 2016 l'ouvrage Mutualiser les ressources documentaires : bibliothèques en réseau), on peut considérer trois axes déterminants dans une documentation durable : mutualiser en 
réseau d'établissements ; mutualiser en partage de contenus ; mutualiser en communauté d'acteurs.

21 Prenant appui sur cette nécessité de mutualisation associée à celle de prévision, une documentation capitalisée pourrait ainsi se concevoir comme :

22 - une documentation préservée, conservée : posant la question des espaces de stockage et de leurs conditions de conservation et notamment celles des anciens documents papier, films et diapositives et microfiches impliquant un transfert des données sur supports numériques avec exemplaires de sauvegarde sans destruction des originaux et duplication des documents originellement numériques, tout en sachant, point épineux, que les clouds actuels sont sans garantie de pérennité ;

23 - une documentation dynamisée (remise au jour) : conservée en tenant compte des nouvelles questions posées aux archives et notamment par l'observation réflexive du travail du traitement documentaire des objets et collections de musée en retrouvant les témoins et traces des expositions passées par exemple. La patrimonialisation de la documentation par elle-même ne peut échapper au mouvement général. C'est aussi une mémoire réactivée car elle redonne valeur et actualité à un ensemble mémoriel susceptible d'intéresser le public et les chercheurs ; faisant de la conservation et de la documentation un sujet d'exposition ;

24 - une documentation augmentée par les substrats et médiums : le numérique, par ses fichiers, a sur ce point prolongé le travail engagé avec le papier et le film (microfilms et microfiches, diapositives, négatifs, positifs, plaques, supports albuminés, aristotypes, papier argentique et autres reproductions, cartes postales). Il permet maintenant de concevoir la complémentarité du numérique et du papier pour l'édition, ce qui suppose encore de comprendre et appliquer une approche de la diversité, de la circulation des genres et des espèces de la reproduction des œuvres et des objets dans le cadre muséal. Il est aussi un enjeu pour la reconnaissance du travail de service public comme en témoigne la préconisation du Guide Data Culture (2013. Rapporteur Camille Domange) où est souligné qu' "en d'autres termes, favoriser et encourager une culture de la collaboration, permettrait de faire naître une économie de la notoriété et de la construction d'une réputation des fonds détenus et/ou produits par les établissements, organismes et services culturels ». 
La Bibliothèque de la Cité de l'Architecture et du Patrimoine à Paris

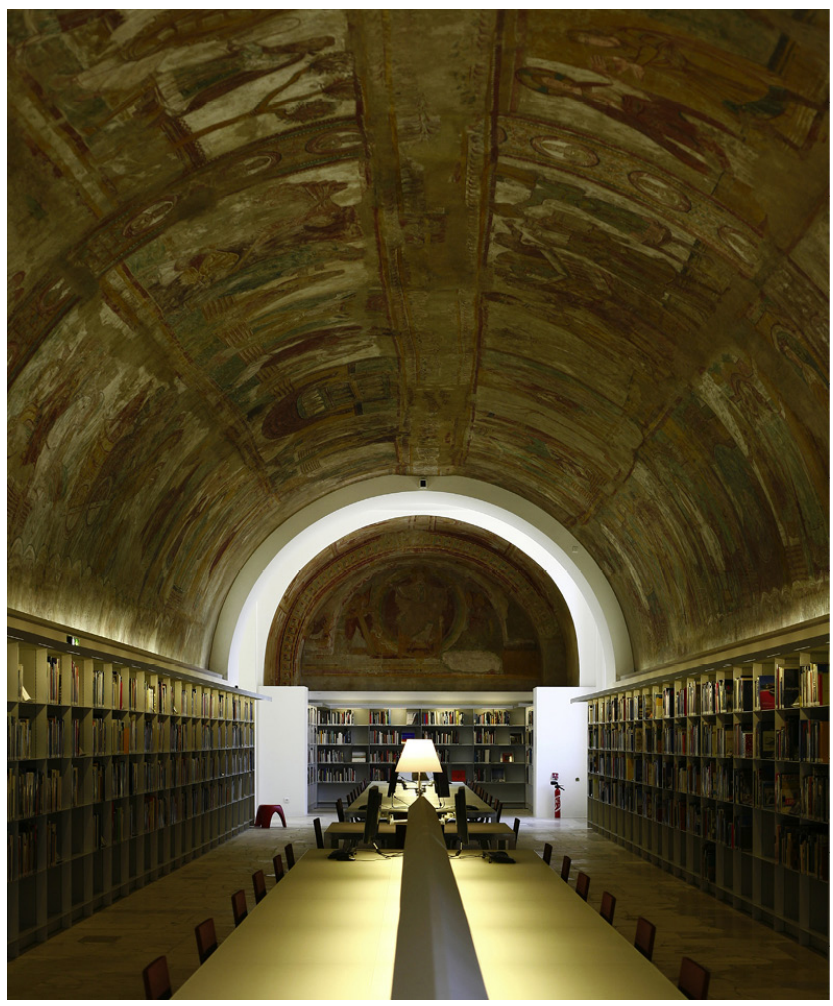

(c) Wikimédia Commons

Le site web du musée du Quai Branly propose une exploration virtuelle de ses collections.
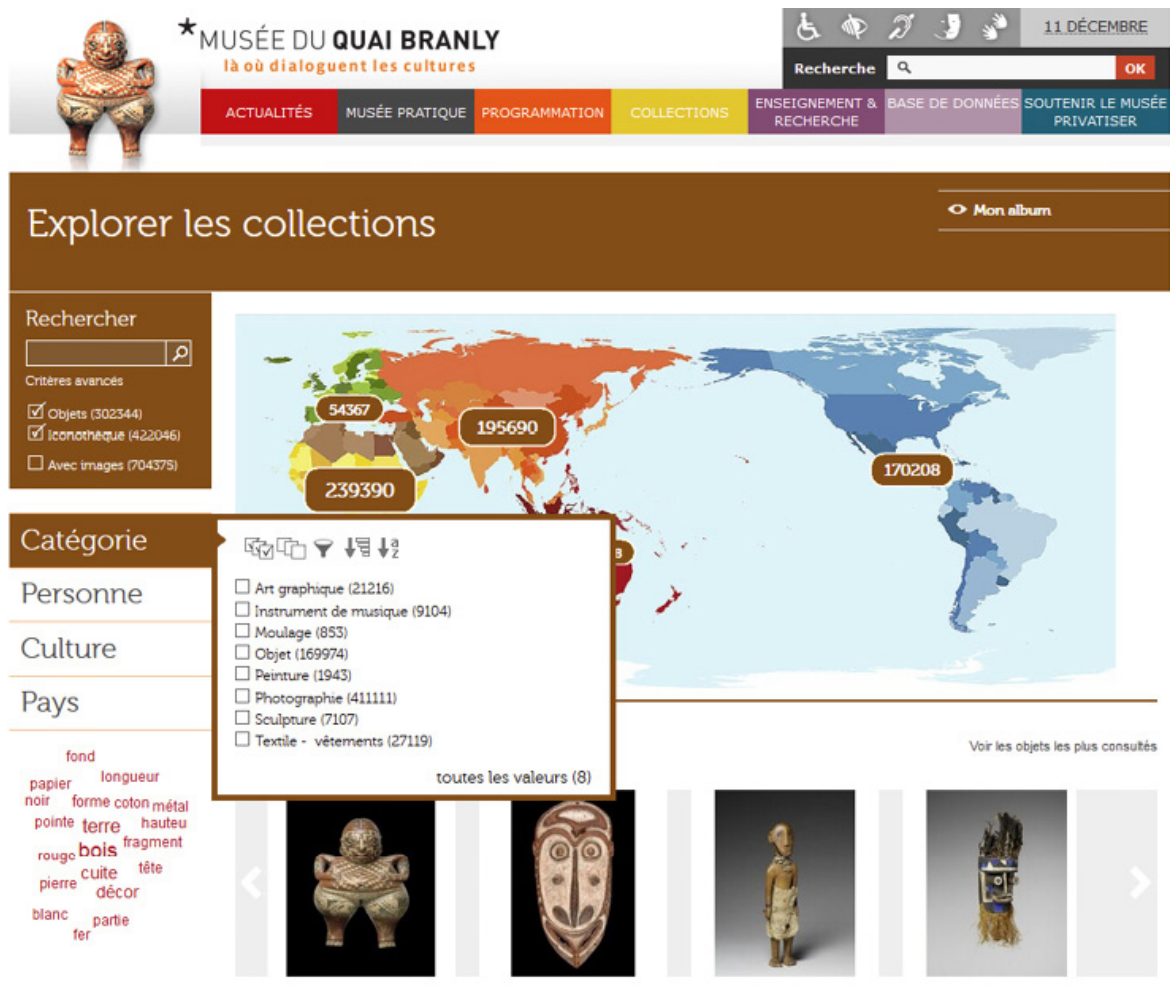

(c) Musée du Quai Branly 


\section{Conclusion} folksonomie interface_fig8_221144133);

\section{BIBLIOGRAPHIE}

Chaumier, S. Les musées : nouvelles formes de médiation, nouveaux projets ? in Bibliothèque nationale de France, actes du colloque Chemins d'accès : L'autonomisation, une école du visiteur? Novembre 2008. En ligne sur www.bnf.fr.

Chevalier, S. Le Réseau Must : mutualiser dans une démarche d'intelligence collective, in Pouchol, J., dir. Mutualiser les pratiques documentaires : bibliothèques en réseau. Villeurbanne : Presses de l'Enssib, 2016. En ligne sur www.enssib.fr.

Grognet, F. Musées manqués, objets perdus ? L’Homme, n¹81, 2007. En ligne sur journals.openedition.org.

Guide Data culture. Pour une stratégie numérique de diffusion et de réutilisation des données publiques numériques du secteur culturel. Rapporteur Domange, C. Ministère de la Culture et de la Communication, Secrétariat général, 2013, 50 p. En ligne sur www.semaphore.culture.gouv.fr.

Lewis, G. Rôle des musées et Code professionnel de déontologie, in Icom, Comment gérer un musée : Manuel pratique. Paris : Unesco, 2006, pp. 1-16. En ligne sur www.icom.museum

Morin, E. La méthode. Les idées (tome 4). Paris : Éditions du Seuil, Coll. Points, 1991, 261 p. 
Pouchol, J. (dir.) Mutualiser les ressources documentaires : bibliothèques en réseau. Villeurbanne : Presses de l'Enssib, La Boîte à outil, 2016, 196 p.

Régimbeau, G. (dir.) Documenter les collections. Cataloguer l'exposition, Culture \& Musées, $\mathrm{n}^{\circ} 22$, 2013, 218 p.

Régimbeau, G. Documents partagés, Documentaliste-Sciences de l'information, $\mathrm{n}^{\circ}$ 2, vol. 51, juin 2014, pp. 44-45. En ligne sur www.cairn.info.

Rizza, M. (dir.) Dossier : Document et musée : du discours sur l'œuvre à la médiation culturelle, Documentaliste-Sciences de l'information, $\mathrm{n}^{\circ} 2$, vol. 51, juin 2014, pp. 28-69. En ligne sur www.cairn.info.

Schnapp, A. Préface, in Picot, N. (dir.) Arts en bibliothèques. Paris : Électre-Éditions du Cercle de la Librairie, 2003.

Sergent, B. Du musée de l'Homme au musée du quai Branly : la régression culturelle, Cahiers d'histoire. Revue d'histoire critique, $n^{\circ} 101,2007$. En ligne sur journals.openedition.org.

\section{NOTES}

1. Sommaire du dossier de Culture \& Musées, $n^{\circ} 22,2013$, « Documenter les collections. Cataloguer l'exposition » : Régimbeau, G. Introduction ; Rizza, M. La numérisation du dossier d'œuvre : enjeux organisationnels pour les musées; Severo, M. La liste du patrimoine mondial : une approche par le système d'information; Després-Lonnet, M. Le patrimoine culturel numérique : entre compilation et computation ; Le Bœuf, P. Le modèle conceptuel de référence du Cidoc : de la sémantique des inventaires aux musées en dialogue ; Giguère, A. Documentation et muséalisation de la performance; Parcollet, R. et Szacka, L.-C. Écrire l'histoire des expositions : réflexions sur la constitution d'un catalogue raisonné d'expositions.

\section{RÉSUMÉS}

L'organisation, le partage, la conservation et la médiation des documents culturels et scientifiques sont les principaux angles envisagés par l'auteur pour traiter du thème de la capitalisation des ressources documentaires dans les institutions muséales.

\section{INDEX}

Mots-clés : Ressources documentaires, épistémologie, conservation

\section{AUTEUR}

\section{GÉRARD RÉGIMBEAU}

Professeur des universités en Sciences de l'information et de la communication, université PaulValéry Montpellier 3 et chercheur au laboratoire Lérass-Céric 
gerard.regimbeau@univ-montp3.fr 